\title{
A cost-effective approach to handle measurement and verification uncertainties of energy savings
}

\author{
Zadok Olinga, Xiaohua Xia, Xianming Ye* \\ Department of Electrical, Electronic and Computer Engineering, University of Pretoria, Pretoria 0002, South Africa
}

\begin{abstract}
Measurement and verification $(\mathrm{M} \& \mathrm{~V})$ function has been adopted in many energy efficiency (EE) programmes to quantify energy savings, identify new energy efficiency opportunities, improve EE programme design, and reduce EE programme performance risks. Budgetary constraints in EE programmes necessitate careful planning to minimise $M \& V$ cost whilst maintaining $M \& V$ accuracy. For this purpose, this study presents an $\mathrm{M} \& \mathrm{~V}$ cost minimisation model to handle $\mathrm{M} \& \mathrm{~V}$ sampling and modelling uncertainties costeffectively. In the proposed model, the objective function is the total $M \& V$ project cost, which consists of $M \& V$ sampling cost, modelling cost, and overhead cost. The required $M \& V$ accuracy in terms of the 90/10 criterion is formulated as the constraints of this optimisation problem. Optimal solutions to the proposed model specify the required baseline model accuracy and sample sizes to achieve the desired $M \& V$ accuracy. The proposed model is applied to design an optimal $M \& V$ sampling and modelling plan for a traffic light retrofit project. Results show that the optimal $M \& V$ plan reduces the sampling cost by $42 \%$ and the total $M \& V$ cost by $11 \%$ against the solutions obtained by partial optimisation.
\end{abstract}

Keywords: $\mathrm{M} \& \mathrm{~V}$, energy efficiency, modelling, uncertainty, optimisation, sampling

\section{Nomenclature}

\section{Variables}

$\lambda_{0}$

$A_{2 n}$

$A_{3 n}$

$A_{4 n}$

$A_{4 r n}$

$a_{i}$

$b_{i}$

$C_{0}$

$C_{m}$

$C_{s}$

${ }^{*}$ Corresponding author. Tel.: +27 (0)12 420 4353; Email address: xianming.ye@up.ac.za (Xianming Ye).

$C V_{i}$

$\mathrm{CVm}$

$C V m_{i}^{*}$ tion model

the quantity of 2-aspect fittings at a traffic intersection

the quantity of 3-aspect fittings at a traffic intersection

the quantity of 4-aspect fittings at a traffic intersection

the quantity of 4-aspect fittings with turning arrows at a traffic intersection

the procurement price per meter in the $i$ th group nomeqref 0

the installation cost per meter in the $i$ th group

the overhead cost

the $\mathrm{M} \& \mathrm{~V}$ modelling cost per model

the metering and sampling cost per $\mathrm{M} \& \mathrm{~V}$ project

Postprint submitted to Energy

$E_{i}$

$G_{n}$ $i$

I

j

$J$

$k$

$n$

$n_{i}$

$n_{i}^{*}$

$N_{i}$

$p$

$R^{2}$

the coefficient of variation of the $i$ th group the $\mathrm{CV}(\mathrm{RMSE})$ of an M\&V baseline model the optimal $\mathrm{CV}(\mathrm{RMSE})$ of an M\&V baseline model the daily energy consumption per traffic intersection of the $i$ th stratum the quantity of green signal lamps at a traffic intersection

$H R_{k} \quad$ the hourly rate of $\mathrm{M} \& \mathrm{~V}$ professionals

the counter of sampling strata

the total number of sampling strata

the counter of baseline models

the total number of baseline models

the professional level of $\mathrm{M} \& \mathrm{~V}$ practitioners

the number of observations

the sample size of the $i$ th stratum

the optimal sample size of the $i$ th stratum

the population size of the $i$ th stratum

the relative precision

the coefficient of determination 


$\begin{array}{ll}R_{n} & \begin{array}{l}\text { the quantity of red signal lamps at a traffic inter- } \\ \text { section }\end{array} \\ T_{k} & \text { the time spent on energy modelling } \\ U_{m} & \text { the total M\&V uncertainty } \\ U_{s} & \text { the total modelling uncertainty } \\ w & \text { the number of independent parameters in the } \\ Y_{n} & \text { baseline model } \\ Y_{i} & \text { the quantity of yellow signal lamps at a traffic } \\ Y_{Y} & \text { the mean of observations } \\ & \text { the value of } Y \text { predicted by regression model } \\ & \text { the sample mean in the } i \text { th stratum }\end{array}$

Abbreviations

ASHRAE American society of heating, refrigeration and air-conditioning engineers

CDM clean development mechanism

CFL compact fluorescent lamp

CV coefficient of variation

ECM energy conservation measure

EEDSM energy efficiency and demand side management

IPMVP international performance measurement and verification protocol

ISO international standards organisation

LED light emitting diode

$\mathrm{M} \& \mathrm{~V} \quad$ measurement and verification

R South African Rand

RMSE root mean square error

SANS South African National Standard

\section{Introduction}

Measurement and Verification $(\mathrm{M} \& \mathrm{~V})$ provides an impartial and replicable process to quantify energy and demand savings in energy efficiency (EE) and demand side management (DSM) projects [16] in more than 15 countries in Europe [36], North America [36], and Asia [30]. Popular M\&V guidelines are provided in the international performance measurement and verification protocol (IPMVP), which gives common practice recommendations and principles for quantifying energy and water savings [16]. Four M\&V options are presented in the IPMVP that can be flexibly applied to determine energy savings from various $M \& V$ projects [16]. Similar and widely referenced $\mathrm{M} \& \mathrm{~V}$ guidelines are given by the American society of heating, refrigeration and air-conditioning engineers (ASHRAE) Guideline 14 [3] and the Federal energy management program (FEMP) [41]. These three guidelines form the basis of other M\&V guidelines such as but not limited to the South African M\&V guideline for the national energy efficiency and demand side management (EEDSM) program [15], the Australian M\&V best practice guideline [4], and the state guidelines in California [37] and Texas [44] of the USA, and Australia [28]. The international standards organisation (ISO) has also released a standard for $\mathrm{M} \& \mathrm{~V}$, which establishes general principles and guidelines for the $M \& V$ process [21].

Reporting energy savings by the $M \& V$ process always includes some degree of uncertainty [41], including both quantifiable and unquantifiable uncertainties. Unquantifiable uncertainties arise from poor meter placement, inaccurate estimates in the IPMVP Option A or mis-estimation of interactive effect$\mathrm{s}$ in the IPMVP Options A or B [16]. There are three types of quantifiable $\mathrm{M} \& \mathrm{~V}$ uncertainties; namely measurement, sampling, and modelling uncertainty. Achieving a higher level of $\mathrm{M} \& \mathrm{~V}$ accuracy by reducing $\mathrm{M} \& \mathrm{~V}$ uncertainties usually implies greater cost [3]. However, M\&V budgets tend to be limited. The IPMVP [16] and FEMP [41] M\&V guidelines recommend that the $\mathrm{M} \& \mathrm{~V}$ cost does not exceed $10 \%$ of the average annual savings being assessed. Other $\mathrm{M} \& \mathrm{~V}$ guidelines such as the $\mathrm{M} \& \mathrm{~V}$ handbook [2] and the California evaluation framework [37] give cost limits based on the IPMVP M\&V Options being used. These cost limits range from a minimum of $1 \%$ of the annual measured savings for the IPMVP Option A to a maximum of $10 \%$ for the IPMVP Option D. Therefore, researchers, $\mathrm{M} \& \mathrm{~V}$ practitioners, and energy efficiency project participants are always eager to find cost-effective solutions to handle the $\mathrm{M} \& \mathrm{~V}$ uncertainties. To achieve this target, relationship between $M \& V$ cost and uncertainty needs to be identified. Existing studies have made positive contribution in $M \& V$ uncertainty and $\mathrm{M} \& \mathrm{~V}$ cost analysis.

The measurement uncertainty in the $M \& V$ process results from instrumentation error due to poor measurement equipment calibration, data tracking errors, and human errors in data capturing [23]. Measurement uncertainty is unavoidable, although it can be mitigated with proper data handling protocols and the use of high-accuracy, calibrated metering equipment such as Class 1, Class 2, and Class 3 meters with specified error bands of $\pm 1.5 \%, \pm 2.5 \%$, and $\pm 4 \%$, respectively as given in Part 21 
[19] and Part 23 [20] of the International Standard 62053 of static meters for reactive energy.

Practically, there are projects with large quantities of EE devices spread over large geographical areas, such as large scale lighting retrofit [47], solar water heater mass roll out [17], and residential rebate programs [24]. Due to budgetary constraints not all devices can be metered, and so sampling is used, which introduces the sampling uncertainties into the reported energy savings [22]. The sampling uncertainty is avoidable for a small population project where meters are applied to the whole population of the involved EE units. The sampling uncertainty can be mitigated through use of sufficient sample sizes [1] and appropriate sampling approaches such as simple random sampling, stratified random sampling, and cluster sampling, to name a few [39]. For instance, to satisfy the $90 / 10$ criterion for clean development mechanism (CDM) projects with the minimal $\mathrm{M} \& \mathrm{~V}$ cost, an optimal sampling plan is developed in [47] that balances sampling uncertainties across different lighting groups with different levels of sampling uncertainties. In addition, longitudinal CDM sampling designs for lighting retrofit projects are proposed in [48] when considering a linear lam$\mathrm{p}$ population decay pattern, and improved longitudinal CDM sampling designs are developed in [9] by incorporating nonlinear lamp population decay characteristics. In order to obtain the best metering and sampling plan for $\mathrm{M} \& \mathrm{~V}$ of largescale lighting projects, three $\mathrm{M} \& \mathrm{~V}$ metering cost minimisation approaches are provided in [46] by using spatial optimisation, longitudinal optimisation, and combined spatial and longitudinal optimisation strategies. These studies achieve cost savings for $\mathrm{M} \& \mathrm{~V}$ projects by applying optimal sample sizes to handle the sampling uncertainty.

Calculating energy savings includes comparing actual energy consumption to the baseline energy consumption at the post-implementation phase of an energy efficiency project. But the energy consumption in the business-as-usual scenario at the post-implementation phase is never measurable as highlighted in [45]. A baseline model is thus needed to predict the energy that would have been consumed had the energy conservation measures not been installed [16]. The energy baseline modelling process thus introduces the modelling uncertainties to the $\mathrm{M} \& \mathrm{~V}$ process. Various modelling techniques are used to characterise the relationship between energy consumption and typical energy driving factors such as temperature, production, and facility occupancy rate, etc. These modelling techniques are but not limited to linear regression [10], support vector machines [12], Gaussian process modelling [7], cross-validation [18], and artificial neural networks [5]. The modelling uncertainty is unavoidable when the baseline model is applied for baseline adjustments. Indeed, modelling uncertainty can be properly handled by ensuring that the right function form is used for modelling and key parameters are included in the models [16].

A number of existing $M \& V$ research articles typically focus on baseline model development. In [10], linear regression modelling is used to develop a baseline model for a conveyor belt retrofit project. The model accuracy is judged using the mean bias error and the coefficient of variation of the rootmean-square error (CV(RMSE)). In the same manner, baseline modelling for building energy consumption is carried out using the whole facility $\mathrm{M} \& \mathrm{~V}$ approach employ multivariate linear regression with the key energy governing factors, such as the outdoor dry bulb temperatures as shown in [13]. And regression analysis techniques are also applied to model separate building energy systems such as lighting, cooling, heating, and general appliances in buildings [14]. Baseline modelling has also been done using the IPMVP option D: calibrated simulation approach by software 'eQuest' to model and simulate the entire building energy consumption [25].

Linear regression is extended in [43] using cross-validation so that the uncertainty in the baseline model can be better estimated. The approach is also advantageous in deciding how much data is needed for baseline estimation. Cross-validation is also used in [18] for the whole building M\&V baseline, which argues that the normalised root-mean-square-error and median absolute relative total error are critical to the consideration of modelling uncertainty in determining energy savings.

The support vector machines approach has been used to forecast building energy consumption in a tropical region [12] and the ordinary least squares approach is used in [26] to evaluate the suitability of empirically-based models for predicting energy performance of centrifugal water chillers with variable chilled water flow. Unlike prevailing modelling approaches that focus on the uncertainties in the baseline model, [32] proposes that baseline models be evaluated in terms of the ratio of expected uncertainty in the savings against the total savings.

Input data uncertainty has been tackled in [7], where the Gaussian process modelling and a Monte Carlo expectation maximisation framework is used to develop baseline energy models that take the input data uncertainty into account. These models have the benefit of reducing $\mathrm{M} \& \mathrm{~V}$ cost by reducing the amount of $\mathrm{M} \& \mathrm{~V}$ data [7]. Additionally, general guidelines for evaluating uncertainty in measured data are provided in [23], which describes a measurement model in the form of a functional relationship between input and output quantities such as current, voltage, and resistance [34].

In the literature, existing studies tend to consider $M \& V$ modelling, sampling, and measurement uncertainties separately. And little efforts have been conducted in revealing the accurate relationship between $\mathrm{M} \& \mathrm{~V}$ modelling cost and uncertainty. Practically, an accurate and reliable M\&V function has become an indispensable guarantee for various energy efficiency programmes such as carbon trading [42], tradable white certificate scheme [6], EEDSM programmes, and performance contracting. In order to handle $\mathrm{M} \& \mathrm{~V}$ uncertainties cost-effectively, this study aims to examine sampling and modelling uncertainties together to minimise $\mathrm{M} \& \mathrm{~V}$ cost by developing an $\mathrm{M} \& \mathrm{~V}$ cost minimisation model. Optimal solutions to the proposed $\mathrm{M} \& \mathrm{~V}$ cost optimisation model specify the required baseline model accuracy and sample sizes to achieve the desired M\&V accuracy. In addition, the model also provides flexibility in designing optimal and easily implementable $M \& V$ plans, which can either apply more accurate baseline models and fewer sample sizes, or less accurate baseline models and greater sample sizes to achieve the same level of $M \& V$ accuracy. This study pays less attention to the measurement uncertainty as it has been noted 
that measurement uncertainty makes a negligible contribution to the overall uncertainty for electricity metering cases where population variance is not unusually low [8]. However, efforts on dealing with sampling and modelling uncertainties are believed to be the most significant contributors to the entire $M \& V$ cost, especially when both the modelling and sampling techniques are used during the $\mathrm{M} \& \mathrm{~V}$ process.

To develop the $\mathrm{M} \& \mathrm{~V}$ cost minimisation model, the total $\mathrm{M} \& \mathrm{~V}$ cost consisting of the $\mathrm{M} \& \mathrm{~V}$ sampling cost, the baseline modelling cost, and the overhead cost is formulated as the objective function. In the objective function, the $M \& V$ baseline model modelling cost is formulated as a function of model accuracy by regression analysis. The sampling cost includes the meter procurement and installation cost, which is directly correlated to the sample size. The required $M \& V$ accuracy in terms of the 90/10 criterion is formulated as the constraints of this optimisation problem. Optimal M\&V modelling and sampling strategies are designed for a traffic light retrofit project as a case study to illustrate the effectiveness of the proposed model. Comparisons between the optimal and non-optimal solutions to the case study show advantageous cost saving performance of the proposed model. More precisely, the optimal solutions reduce the sampling cost by $42 \%$ and the total M\&V cost by $11 \%$ against the solutions obtained by optimal modelling but nonoptimal sampling solutions. In addition, flexibilities in designing easily implementable $\mathrm{M} \& \mathrm{~V}$ modelling and sampling plans of the proposed model are also revealed by two simulations. The simulation results show that the proposed model is able to offer flexible trade-offs between the modelling and sampling uncertainties; namely, using more accurate baseline models and fewer sample sizes or less accurate baseline models but greater sample sizes to achieve the same level of $M \& V$ accuracy. Major contributions of this study are as follows: 1) the $M \& V$ baseline model cost is formulated as a function of its model accuracy, which enables quantitative analysis between the M\&V baseline modelling accuracy and cost; and 2) an M\&V cost minimisation model is proposed to handle both the $\mathrm{M} \& \mathrm{~V}$ modelling and sampling uncertainties cost-effectively. The effectiveness and flexibility of this model are demonstrated by a case study and the simulation results.

The rest of this paper is organised as follows: in Section 2, a $M \& V$ cost minimisation model is developed with a detailed analysis of the combined sampling and modelling uncertainties, and the M\&V cost analysis and modelling. Section 3 presents a case study to illustrate the effectiveness of the proposed M\&V cost minimisation model. A sensitivity analysis on the proposed model is carried out in Section 4 followed by a discussion. Conclusions are summarised in Section 5.

\section{Problem formulation}

In this section, an $\mathrm{M} \& \mathrm{~V}$ cost minimisation model is developed to design optimal M\&V plans to handle the M\&V modelling and sampling uncertainties cost-effectively. For this purpose, typical M\&V cost factors are reviewed and a model is developed to characterise the relationship between the $\mathrm{M} \& \mathrm{~V}$ modelling cost and model accuracy. In addition, the formulation of combined $\mathrm{M} \& \mathrm{~V}$ uncertainties including both the modelling and sampling uncertainties is introduced under different practical scenarios. Based on the M\&V cost and modelling analysis, an $\mathrm{M} \& \mathrm{~V}$ cost minimisation model is developed. Objective function of the model includes the M\&V sampling, modelling, and overhead costs, and the M\&V accuracy requirements are formulated as the constraints of the optimisation model.

\subsection{MEVV cost analysis}

In many energy efficiency programmes [11], M\&V is an indispensable process to quantify energy savings achieved by the implemented energy conservation measures. In practice, there are cost implications of the M\&V service, including energy audit, metering, sampling, data analysis, and energy savings reporting, etc. From the engineering perspective, numerous factors are affecting the $\mathrm{M} \& \mathrm{~V}$ cost while the major ones are:

- amount and complexity of measurement equipment;

- sample sizes used to sample representative items;

- amount of engineering required to develop the M\&V plan;

- number and complexity of independent variables that are accounted for in baseline models;

- miscellaneous $\mathrm{M} \& \mathrm{~V}$ related activities such as travelling for site visit, communication, reporting, documentation, and management; and

- $\mathrm{M} \& \mathrm{~V}$ accuracy requirements.

In general, most of the above-mentioned $\mathrm{M} \& \mathrm{~V}$ cost factors are directly related to the accuracy of $M \& V$ reporting. The $\mathrm{M} \& \mathrm{~V}$ accuracy is usually expressed as quantifiable uncertainties of final $M \& V$ outcomes, such as energy/demand savings or cost savings. The quantifiable $M \& V$ uncertainties refer to the $\mathrm{M} \& \mathrm{~V}$ measurement, sampling, and modelling uncertainties as categorised in [16]. In line with this, the $M \& V$ cost can be generally classified into metering cost, sampling cost, modelling cost, and overhead cost. The metering cost normally includes the meter procurement, installation, commissioning, and calibration cost. The amount of metering budget is decided by the required meter device specifications and service cost for installation and calibration [29]. Sampling cost is directly related to the sample size to achieve a better sampling accuracy where a greater sample size usually implies a higher sampling cost. The modelling cost is generally more difficult to decide but it is linked to the level of M\&V professionals appointed, and the time that the $\mathrm{M} \& \mathrm{~V}$ professionals spend on data processing, model construction, parameter identification, and model validation to achieve an acceptable level of model accuracy. The overhead cost is usually basic charge for $\mathrm{M} \& \mathrm{~V}$ that includes travelling, reporting, communication, documentation, and management. 
The M\&V metering and sampling cost analysis is given as follows. Let $a_{i}$ denote the procurement $\operatorname{cost}^{1}$ and $b_{i}$ represen$\mathrm{t}$ the installation, calibration, and commission cost for the $i$ th type of metering device to be used for $\mathrm{M} \& \mathrm{~V} . n_{i}$ denotes the number of metering devices used, also known as the sample size for measurement. ${ }^{2}$ Then the metering and sampling cost $C_{s}$ is calculated by Equation (1),

$$
C_{s}=\sum_{i=1}^{I}\left(a_{i}+b_{i}\right) n_{i} .
$$

\subsection{Cost analysis for MEVV baseline modelling}

As discussed, the M\&V modelling cost has several determinants such as the level of expertise from $M \& V$ professionals, the complexities on model construction and parameter identification, and required modelling accuracy. As no previous studies have addressed the cost analysis for M\&V baseline modelling, this study attempts to develop a data-driven model by regression analysis to characterise the relationship between the $\mathrm{M} \& \mathrm{~V}$ modelling cost and model accuracy.

As suggested from both the theoretical aspects [31] and the practical aspects [33] for the baseline modelling methodology, developing a data-driven model for $\mathrm{M} \& \mathrm{~V}$ baseline comprises of following main stages, namely 1) data collection; 2) regression analysis to different mathematic forms with model parameter identification; and 3) to choose the best-fit model according to model $R^{2}$ and CV(RMSE). As introduced in [13],

$$
\begin{gathered}
R^{2}=\frac{\sum_{j=1}^{n}\left(\hat{Y}_{j}-\bar{Y}\right)}{\sum_{j=1}^{n}\left(Y_{j}-\bar{Y}\right)}, \\
C V(R M S E)=\frac{R M S E}{\bar{Y}},
\end{gathered}
$$

and

$$
R M S E=\sqrt{\frac{\sum_{j=1}^{n}\left(Y_{j}-\hat{Y}_{j}\right)^{2}}{n-w}},
$$

where $\bar{Y}$ is the mean of observations $Y_{j} ; \hat{Y}$ is the value of $Y$ predicted by regression model; $n$ is the number of observations; and $w$ is the number of independent parameters in the baseline model. According to the suggestions from both the theoretical aspects [31] and the practical aspects [33] for the baseline modelling methodology, the best-fit model is chosen by the criteria: 1) the highest $R^{2}$ and lowest $\left.\mathrm{CV}(\mathrm{RMSE}) ; 2\right)$ if the $R^{2}$ are at similar range, then the $\mathrm{CV}(\mathrm{RMSE})$ to be given more consideration. Note that the $\mathrm{CV}$ (RMSE) is positive and normalised against the mean of all data observations in this study, which enables comparison between dataset or models with different units and scales.

\footnotetext{
${ }^{1}$ Cost analysis in this study uses South African currency, Rand, and R is short for the Rand.

${ }^{2} i$ is used as a counter of types of metering instruments, which is also used later in this study to denote the counter of stratum for stratified sampling, since we assume one type of metering device to be used in one stratum due to the homogeneity property in each stratum.
}

In this study, the data used for the M\&V modelling cost are obtained from a SANAS Accredited M\&V inspection body, who has provided professional M\&V services to over 300 energy efficiency projects in the national EEDSM programme and tax incentive scheme [27]. As informed by the M\&V inspection body, the M\&V modelling cost is decided by accounting the time spent on the M\&V modelling by different levels of $\mathrm{M} \& \mathrm{~V}$ professionals. More precisely, the M\&V professionals in the company are rated and ranked into four levels, namely M\&V Team Leader, Senior M\&V Engineer, M\&V Engineer, and $\mathrm{M} \& \mathrm{~V}$ Technician according to their qualification and experiences in energy, engineering, and the $\mathrm{M} \& \mathrm{~V}$ industry. Hourly rate of the $\mathrm{M} \& \mathrm{~V}$ professionals are denoted by $H R_{k}, k=1,2,3$, and 4 for the M\&V Team Leader, Senior M\&V Engineer, M\&V Engineer, and M\&V Technician, respectively. For each M\&V project, the time spent on the baseline modelling and uncertainty analysis from different $\mathrm{M} \& \mathrm{~V}$ professionals are recorded as $T_{k}$. The M\&V modelling cost $C$ for each project is calculated by

$$
C=\sum_{k=1}^{4} H R_{k} T_{k}
$$

To protect its business, the M\&V company does not provide detailed $M \& V$ quotations for investigation. Instead, the $M \& V$ company provides a guideline on costing for $\mathrm{M} \& \mathrm{~V}$ modelling, which is summarised in Table 1.

\begin{tabular}{rr} 
Table 1: M\&V modelling cost vs CV(RMSE) \\
\hline CV(RMSE) & Modelling cost (Rand) \\
\hline$(0,5 \%]$ & R 223750 \\
$(5 \%, 10 \%]$ & R 155500 \\
$(10 \%, 15 \%]$ & R 130500 \\
$(15 \%, 20 \%]$ & R 103000 \\
$(20 \%, 25 \%]$ & R 92000 \\
$(25 \%, 30 \%]$ & R 74750 \\
\hline
\end{tabular}

As seen in Table 1 , the M\&V company has different prices for the baseline models with different accuracy levels, which are quantified by $\mathrm{CV}(\mathrm{RMSE})$. The modelling cost summarised in Table 1 are obtained from their historical records on the time spent for the $\mathrm{M} \& \mathrm{~V}$ modelling from $\mathrm{M} \& \mathrm{~V}$ professionals in various $\mathrm{M} \& \mathrm{~V}$ projects. The $\mathrm{M} \& \mathrm{~V}$ company follows [16] as their service standard, which further requires that each baseline model must have a minimum $R^{2}$ of 0.75 , and a worst $\mathrm{CV}(\mathrm{RMSE})$ of $30 \%$.

The data in Table 1 can be expressed as a piecewise function to describe the relationship between the cost and accuracy of an $\mathrm{M} \& \mathrm{~V}$ baseline model. The piecewise function is sufficient to offer guidance to develop $\mathrm{M} \& \mathrm{~V}$ quotations. In order to identify more closer relationship between the cost and accuracy of the $M \& V$ energy modelling, this study aims to developed a data-driven model by regression analysis of the data in Table 1. The regression analysis can be conducted by Matlab or Microsoft Excel tools. During the regression analysis, the maximum CV(RMSE) values are used for each price category in Table 1. Based on these data, a number of data-driven models with different mathematic forms are established. And goodness-of-fit in terms of CV(RMSE) and $R^{2}$ is calculated as 
well during the modelling process, which is provided in Table 2. It is observed that the power model in the form of

$$
C_{m}=601040 C \mathrm{Vm}^{-0.591},
$$

has the lowest $\mathrm{CV}(\mathrm{RMSE})$ and highest $R^{2}$ values among four other mathematical forms namely linear regression, exponential regression, polynomial regression, and hyperbolic regression. In Eq. (6), $C V m$ denotes the CV(RMSE) of an M\&V baseline model while $C_{m}$ denotes the $\mathrm{M} \& \mathrm{~V}$ modelling cost for one baseline model. The chosen power model during the regression analysis is also presented graphically in Figure 1, in which the red markers represent the data used for modelling.

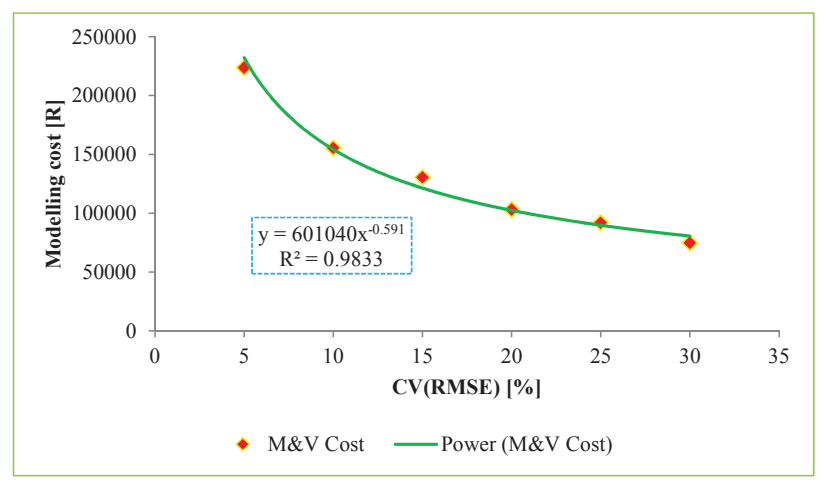

Figure 1: Regression analysis for M\&V modelling cost.

Now the power model is selected to characterise the relationship between M\&V modelling cost and accuracy in this study. Although the selected power model has a very low CV(RMSE) and high $R^{2}$, it has following practical limitations: 1) the data source is obtained from a professional guideline on the costing of $\mathrm{M} \& \mathrm{~V}$ baseline energy modelling. It is believed that better M\&V modelling cost function can be developed when more detailed data such as original baseline modelling quotations, actual hourly rate of $\mathrm{M} \& \mathrm{~V}$ professionals, records of time spent on $\mathrm{M} \& \mathrm{~V}$ modelling, etc. are made available; 2 ) the selected power model does take consideration of market influence such as inflation and price competition. Despite of these disadvantages, the power model for $\mathrm{M} \& \mathrm{~V}$ baseline modelling cost formulation has its value in following aspects: 1) it is the first model that enables quantification of the $\mathrm{M} \& \mathrm{~V}$ baseline model cost and the model accuracy; 2) it is able to provide a guidance on the analysis of $\mathrm{M} \& \mathrm{~V}$ baseline modelling cost and accuracy to $\mathrm{M} \& \mathrm{~V}$ practitioners.

Table 2: Regression models of M\&V baseline cost.

\begin{tabular}{llrc}
\hline Model Type & Function & CV(RMSE) & $R^{2}$ \\
\hline Linear & $y=-5502.9 x+226217$ & $12.96 \%$ & 0.9034 \\
Exponential & $y=251937 e^{-0.042 x}$ & $8.11 \%$ & 0.9749 \\
Polynomial & $y=222.14 x^{2}-13278 x+278050$ & $5.61 \%$ & 0.9819 \\
Power & $y=601040 x^{-0.591}$ & $4.83 \%$ & 0.9833 \\
Hyperbolic & $y=848441.1 \frac{1}{x}+60627.06$ & $7.91 \%$ & 0.9640 \\
\hline
\end{tabular}

\subsection{MEVV uncertainty analysis}

This study focuses on dealing with the modelling and sampling uncertainties in the process of measuring and verifying energy savings, as measurement uncertainties are negligible when sophisticated measurement equipment is adopted. As introduced in the IPMVP, the three types of uncertainties in M\&V can be combined either in an additive or multiplicative way provided that they are independent. Under this assumption, the IPMVP [16] also quantifies the combined uncertainty $U$ of the sampling and modelling uncertainty as

$$
U=\sqrt{U m^{2}+U s^{2}}
$$

where $U m$ and $U s$ denotes the modelling and sampling uncertainties, respectively.

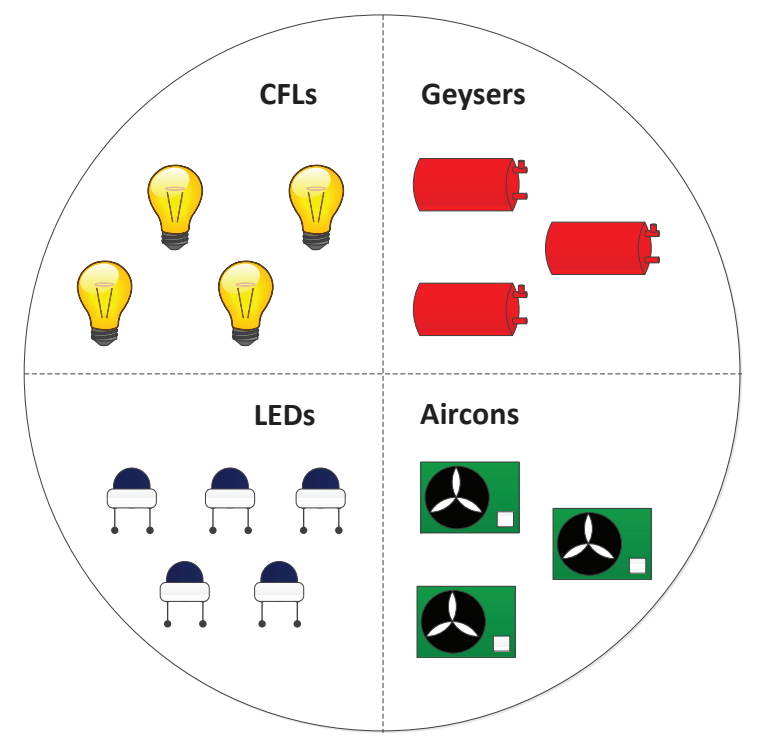

Figure 2: Illustration of combined uncertainty analysis.

Practically, different combinations of uncertainties may exist in an M\&V project. As shown in Figure 2, a project aims to replace all lighting, water heating, and air-conditioning systems with more energy efficient ones in a commercial building. For $\mathrm{M} \& \mathrm{~V}$ purpose, the $\mathrm{M} \& \mathrm{~V}$ practitioners can either use the whole facility approach or the retrofit isolation approach to measure the energy baseline for energy savings determination [16]. When the whole facility approach is adopted to measure the energy consumption of all the lighting, water heating and air-conditioning systems for a whole building baseline energy modelling, there is no sampling uncertainty apply but must only consider the modelling uncertainty. When applying the retrofit isolation approach, sampling techniques will be required if not all devices are measured. In addition, baseline energy models will then be developed for M\&V purpose at the appliance level. In this case, both the modelling and sampling uncertainties must be considered. In the following, the M\&V sampling uncertainty and modelling uncertainty are analysed separately and as a combination for the total $\mathrm{M} \& \mathrm{~V}$ uncertainty analysis.

1) Sampling uncertainty analysis. Given a large-scale lighting retrofit project that includes both CFL and LED technologies as shown in Figure 2, the modelling uncertainty is negligible as the lighting energy consumption is well 
characterised by the product of lighting input wattage and usage time. However, sampling uncertainty need$\mathrm{s}$ to be handled if not all lighting population is measured. In the previous study [47], sampling uncertainties can be cost-effectively handled by applying a stratified sampling approach. The stratified random sampling is a sampling approach to take simple random samples from sub-populations when there are obvious grouping of population elements whose characteristics are more similar within subgroups than across subgroups [40]. The subpopulations are called strata. In [47], the lighting population is firstly stratified into different strata by the coefficient of variation $(\mathrm{CV})$ of the energy usage of individual lamps. The optimal sample size can then be assigned to each stratum for sampling. In this scenario, sampling uncertainty at project level will be a combination of the sampling uncertainties across different lighting strata.

The sampling uncertainty in the $i$ th lighting stratum is described by the sample standard error and is defined as

$$
U s_{i}=\frac{c v_{i} \bar{Y}_{i}}{\sqrt{n_{i}}},
$$

where $c v_{i}$ and $n_{i}$ denotes the $\mathrm{CV}$ and required sample size in the $i$ th lighting stratum, respectively; and $\bar{Y}_{i}$ denotes the sample mean. The sampling uncertainty across each lighting stratum is expressed as

$$
U s=\sqrt{\sum_{i=1}^{I}\left(\frac{N_{i}}{N}\right)^{2} \cdot \frac{\left(c v_{i} \bar{Y}_{i}\right)^{2}}{n_{i}}}
$$

where $I$ is the total number of the lighting strata; $N_{i}$ is the population size of the $i$ th stratum; and $N$ is the total population size.

2) Combined modelling uncertainty analysis. Given a smal1 scale energy conservation project that aims to improve the energy efficiency of several water heaters and air conditioners, sampling uncertainties do not exist when each device is measured for $\mathrm{M} \& \mathrm{~V}$. However, energy baseline models need to be established to adjust the baseline under post-retrofit condition for the savings determination. For projects with both water heaters and air conditioners involved, the baseline model can be designed as a function to describe the relationship between the total energy usage and heating or cooling degree days, or other energy governing factors over the reporting period [13]. Alternatively, baseline energy models can be developed separately for the water heaters and the air conditioners. When two or more baseline energy models are applied in one $\mathrm{M} \& \mathrm{~V}$ project, the modelling uncertainty is quantified as a combination of each baseline energy model uncertainty.

Given an M\&V project with $J$ baseline energy models, uncertainty for each model is formulated as

$$
U m_{j}=C V m_{j} \bar{Y}_{j}
$$

where $\bar{Y}_{j}$ is the average baseline energy consumption and $C V m_{j}$ is the CV(RMSE) of the $j$ th baseline energy model. The combined modelling uncertainty is given by

$$
U m=\sqrt{\sum_{j=1}^{J}\left(\frac{N_{i}}{N}\right)^{2} \cdot U m_{j}^{2}}
$$

where $J$ is the total number of baseline energy models.

3) Combined modelling and sampling uncertainty analysis. At the $\mathrm{M} \& \mathrm{~V}$ project level, the total uncertainty is the combination of the measurement, sampling, and modelling uncertainty. Based on the assumption that measurement uncertainties are negligible, the total project uncertainty including both the sampling and modelling uncertainties are calculated by Eq. (7), and $U s$ and $U m$ in Eq. (7) are obtained by Eq. (9) and Eq. (11), respectively.

\subsection{MEVV cost minimisation model}

In this subsection, an $\mathrm{M} \& \mathrm{~V}$ cost minimisation model is developed to handle both the $\mathrm{M} \& \mathrm{~V}$ sampling and modelling uncertainties cost-effectively. The optimisation objective is to achieve the desired $M \& V$ accuracy with minimal $M \& V$ cost. As introduced in Subsection 2.1, the M\&V cost includes metering and sampling cost, modelling cost, and overhead cost. The $\mathrm{M} \& \mathrm{~V}$ accuracy is defined in terms of the combined modelling and sampling accuracy, which is set to meet the 90/10 criterion in this study. As introduced in [16], the relationship between the 90/10 criterion accuracy and the combined sampling and modelling uncertainty is charactised by

$$
p=\frac{z \times U}{\bar{Y}}
$$

where $p$ is the relative precision and $z$ is the $z$ score related to a confidence level [16].

Let an M\&V project have $I$ sampling strata and $J$ models for the baseline adjustment, it is expected to find the optimal sample size $n_{i}^{*}$ in each sampling stratum and the optimal accuracy level $C V m_{i}^{*}$ for each baseline model that achieves the desired M\&V accuracy with the minimal M\&V cost. This is an optimisation problem that aims to find the optimal solutions $\lambda=\left(C V m_{1}^{*}, \ldots, C V m_{J}^{*}, n_{1}^{*}, \ldots, n_{I}^{*}\right)$, which minimises the overall $\mathrm{M} \& \mathrm{~V} \operatorname{cost} f(\lambda)$

$$
f(\lambda)=\sum_{j=1}^{J} 601040 C V m_{j}^{-0.591}+\sum_{i=1}^{I}\left(a_{i}+b_{i}\right) n_{i}+C_{0}
$$

where $C_{0}$ denotes the overhead cost. The objective function is subject to the constraints

$$
p=\frac{z \times U}{\bar{Y}} \leq 10 \%
$$

where $U$ is the total uncertainty that is calculated by the Eqs. (7)-(11). 


\section{Case study}

In this section, a traffic light retrofit project that has been implemented in South Africa is used to illustrate the effectiveness of the proposed M\&V cost minimisation model in handling both the M\&V modelling and sampling uncertainties costeffectively.

\subsection{Project background}

A traffic light retrofit project has been implemented in several municipalities in South Africa that replaces $56 \mathrm{~W}$ incandescent signal lamps with an equal number of $15 \mathrm{~W}$ LED signal lights. A number of 2200 traffic intersections have been retrofitted by more than 125000 LED signal lamps. Due to different conditions of existing traffic light systems, this project includes two types of lighting retrofit activities, traffic light retrofits and traffic set retrofits. In 1320 traffic intersections, the $56 \mathrm{~W}$ signal lamps, which include red, amber, and green coloured ones, are replaced by the new $15 \mathrm{~W}$ LED lights within a traffic light set. In the remaining 880 traffic intersections, the whole traffic light sets including 4-aspect, 3-aspect, and 2-aspect ones are replaced by new ones with LED technologies. It is required by local government that the energy savings of this project must be assessed by the $\mathrm{M} \& \mathrm{~V}$ process.

\subsection{MEVV approach for the case study}

In order to reliably quantify the energy savings for this traffic light retrofit project, the project boundary, metering and sampling plan, baseline calculation and baseline adjustment approaches need to be specifically designed.

As introduced in the Subsection 3.1, the project boundary includes all the 200 traffic intersections and all the LED signal lamps. For the $\mathrm{M} \& \mathrm{~V}$ purpose, it is applicable to measure the daily energy consumption either in terms of the traffic intersections or individual LED signal lamps. In order to reduce the sampling population, which will consequently reduce the sample size and sampling cost, it is decided that the energy consumption per traffic intersection will be measured. Therefore, the IPMVP Option C: the whole facility measurement approach is applied to this $M \& V$ case study.

As introduced in [35], daily energy consumption of traffic intersections varies in terms of different operation schedules and lamp quantities. Given the two different traffic light retrofit activities, the energy consumptions are randomly sampled and recorded for both types of retrofits at various traffic intersections over a short period. Based on metered data gathered during test sampling, it is found that the average energy consumption of the intersections with individual signal lamp retrofits is 1.91 $\mathrm{kWh}$ with a standard deviation of $0.382 \mathrm{kWh}$, while the average energy consumption of intersections with traffic set retrofits is $1.415 \mathrm{kWh}$ with a standard deviation of $0.708 \mathrm{kWh}$. According to the test sampling, the sampling $\mathrm{CV}$ of the daily energy consumption of the 1320 intersections is less than 0.2 while the $\mathrm{CV}$ of the 880 traffic lamp set retrofit intersections is taken as 0.5 . The traffic intersections are classified into two groups; namely, Groups I and II for stratified sampling according to the different sampling CV values of each stratum. Optimal sample sizes will be decided by the model (13)-(14). The same sample size will be used at both the baseline and post-retrofit periods. Given different uncertainty levels of the daily energy consumption, meters with different functions and prices will be installed at randomly selected intersections from each stratum. The price for the meter procurement and installations can be obtained from meter suppliers.

There are two traffic light baseline modelling approaches introduced in [35] as shown in Equations (15)-(16). The daily energy consumption per intersection can be formulated as the quantity of each type of signal lamp in terms of different lamp colours. For instance, the daily energy consumption per intersection $E_{1}$ can be denoted by

$$
E_{1}=\beta_{0}+\beta_{1} R_{n}+\beta_{2} Y_{n}+\beta_{3} G_{n},
$$

where $\beta_{0}, \beta_{1}, \beta_{2}$, and $\beta_{3}$ are regression coefficients; $R_{n}, Y_{n}$, and $G_{n}$ are the quantity of Red, Yellow, and Green signal lamps, respectively. Alternatively, the energy consumption for the traffic set retrofit is given as

$$
E_{2}=\alpha_{0}+\alpha_{1} A_{2 n}+\alpha_{2} A_{3 n}+\alpha_{3} A_{4 n}+\alpha_{4} A_{r n},
$$

where $\alpha_{0}, \alpha_{1}, \alpha_{2}, \alpha_{3}$ and $\alpha_{4}$ are regression coefficients; $A_{2 n}$ and $A_{3 n}$ denote the quantity of 2-aspect and 3-aspect traffic light sets, respectively; $A_{4 n}$ and $A_{r n}$ denotes the quantity of the 4-aspect fittings with pedestrian signals, and 4-aspect fittings with turning arrows, respectively.

The baseline energy consumption will be the aggregated energy consumption of all the intersections in the project multiplied by the number of days in the baseline measurement period. The two baseline models (15)-(16) will be applied for baseline adjustments under the post-retrofit period.

\subsection{Optimal MEVV plan for the case study}

In order to design an cost-effectively $M \& V$ plan for this case study, M\&V professionals need to solve the proposed optimisation model (13)-(14) with the project specific information, which are summarised in Table 3. To demonstrate the effectiveness of the proposed optimisation approach, solutions without optimisation are calculated as a benchmark for comparison. As mentioned in the introduction section, there is no existing study that has a cost analysis in dealing with M\&V modelling uncertainties. In the absence of a direct benchmark to the study, it is proposed that the optimal solutions be compared with the partially optimised solutions, in order to highlight the effectiveness of the proposed $\mathrm{M} \& \mathrm{~V}$ cost minimisation model. The partial optimal solutions (POS) are obtained by

\section{POS1:Optimal modelling but non-optimal sampling ap-} proach. In this approach, the optimal modelling accuracy is assigned but the sample sizes are not optimised and calculated by the sample size determination formula as given in [38],

$$
n_{0}=\frac{z^{2} c v^{2}}{p^{2}} .
$$


Table 3: Initial values for the case study.

\begin{tabular}{lcc}
\hline Parameters & Group I & Group II \\
\hline Meter unit price & $a_{1}=\mathrm{R} 500$ & $a_{2}=\mathrm{R} \mathrm{1500}$ \\
Installation per meter & $b_{1}=\mathrm{R} \mathrm{195}$ & $b_{2}=\mathrm{R} \mathrm{320}$ \\
CV values & $c v_{1}=0.20$ & $c v_{2}=0.50$ \\
Estimated $\bar{Y}_{i}$ & $\bar{Y}_{1}=1.91 \mathrm{kWh}$ & $\bar{Y}_{2}=1.415 \mathrm{kWh}$ \\
Population & $N_{1}=1320$ & $N_{2}=880$ \\
\hline
\end{tabular}

Table 4: Solutions to the case study with optimal modelling only.

\begin{tabular}{lccc}
\hline Parameters & Group I & Group II & Overall \\
\hline Optimal CV(RMSE) & $5.356 \%$ & $9.233 \%$ & $4.710 \%$ \\
Non-optimal sample size & 11 & 68 & 79 \\
Sampling cost & R 7 645 & R 123 760 & R 131 405 \\
Modelling cost & R 222 920 & R 161 580 & R 384 500 \\
Project cost & R 230 565 & R 285 340 & R 515 905 \\
\hline
\end{tabular}

POS2:Optimal sampling but non-optimal modelling approach. In this approach, the optimal sample sizes are designed but the model accuracy is not optimised. The ASHRAE M\&V guidelines [3] recommends that the IPMVP: Option $\mathrm{C}$ baseline models should have a poorest CV(RMSE) of $25 \%$. Thus the CV(RMSE) of $25 \%$ is chosen in this approach to establish the benchmark.

The initial values in Table 3 are used to calculate both the optimal and partial optimal solutions. The results for POS1 and POS2 are presented in Tables 4-5.

To obtain the optimal solutions, the $\mathrm{M} \& \mathrm{~V}$ cost minimisation model in Eqs. (13)-(14) is solved using the case study specific information given in Table 3. The optimisation problem in Eqs. (13)-(14) is a non-linear problem and it is solved using "MATLAB" simulation software, specifically the fmincon optimisation function. The following settings are employed for the optimisation function: the tolerance on the function value, tolfun, the tolerance on the constraints, tolcon, and the termination tolerance on the design variables, tolx are all set to $10^{-45}$. The optimal sample sizes are integers which are obtained using integer programming algorithms. Because this study deals with the practical problem of minimising $\mathrm{M} \& \mathrm{~V}$ project cost, real-valued sample sizes are used for the optimisation. Once the optimal solution is found, the ceil function is applied to the sample size to obtain the rounded integer sample sizes. Mathematically, the rounded sample sizes are only sub-optimal solutions. Henceforth, the terms optimal/optimise and minimal/minimise refer to the rounded sub-optimal solutions. The starting point of the optimisation is arbitrarily chosen as $\lambda_{0}=(35,100,50,50)$. With lower bounds $l b=(0,0,0,0)$ and upper bounds $u b=(\infty, \infty, \infty$, $\infty)$. The optimal solutions to the case study are given in Table 6 .

In Tables 4-6, the overall modelling CV(RMSE) is calculated by the total modelling uncertainty $U_{m}$ over the weighted aver-

Table 5: Solutions to the case study with optimal sampling only.

\begin{tabular}{lccc}
\hline Parameters & Group I & Group II & Overall \\
\hline Non-optimal CV(RMSE) & $25 \%$ & $25 \%$ & $18.67 \%$ \\
Optimal sample size & 37 & 28 & 65 \\
Sampling cost & R 25 715 & R 50 960 & R 76 675 \\
Modelling cost & R 89685 & R 89685 & R 179 370 \\
Project cost & R 115 400 & R 140645 & R 256 045 \\
\hline
\end{tabular}

\begin{tabular}{lccc}
\multicolumn{4}{c}{ Table 6: Optimal solutions to the case study. } \\
\hline Parameters & Group I & Group II & Overall \\
\hline Optimal CV(RMSE) & $5.356 \%$ & $9.233 \%$ & $4.710 \%$ \\
Optimal sample size & 37 & 28 & 65 \\
Sampling cost & R 25 715 & R 50 960 & R 76 675 \\
Modelling cost & R 222 920 & R 161 580 & R 384 500 \\
Project cost & R 248 635 & R 212 540 & R 461 175 \\
\hline
\end{tabular}

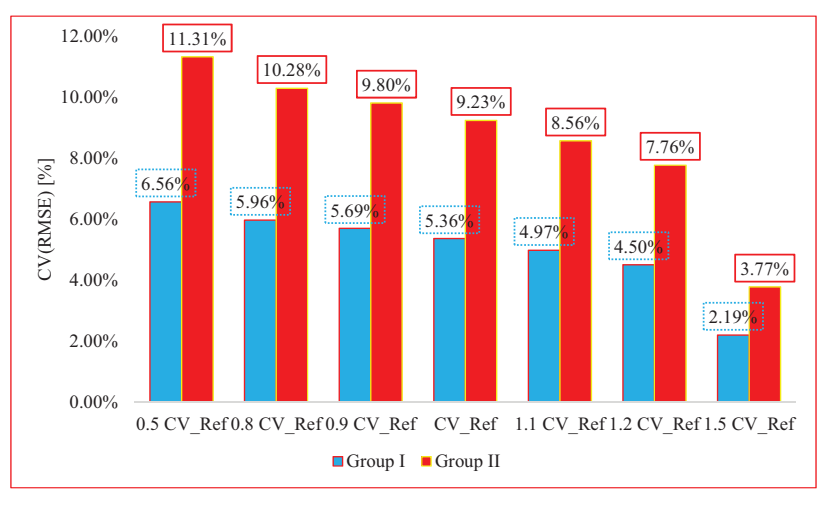

Figure 3: CV(RMSE) when sampling uncertainties change.

age daily energy consumption $\bar{Y}$. When comparing the results in Table 4 and Table 6 , the optimal solutions reduce the sampling cost by $42 \%$ and the total M\&V cost by $11 \%$ against the solutions obtained by the POS1. The results given in Table 5 offers a lower $\mathrm{M} \& \mathrm{~V}$ cost than the optimal solution. However, as the model accuracy in Table 5 is much lower than the optimal model accuracy, the solutions in Table 5 cannot satisfy the required 90/10 criterion for the $M \& V$ reporting.

\section{Model analysis and discussion}

The optimal solutions to the case study in Section 3 illustrate the advantageous performance of the proposed M\&V cost optimisation model in designing an optimal M\&V plan for a specific traffic light retrofit $M \& V$ project. In order to test the applicability and flexibility of the proposed model for the cost-effective design of similar traffic light projects, simulations have been carried out to evaluate the model performance when applying the model to $M \& V$ projects with different characteristics. In addition, the simulations also aim to demonstrate flexibility of the proposed optimisation model in designing optimal $\mathrm{M} \& \mathrm{~V}$ plan. For instance, some possible optimal solutions may require very high modelling accuracy with relatively low requirements on sampling accuracy, which are not easily implementable. In this case, it is expected that more easily implementable optimal solutions, i.e., a lower modelling accuracy with greater sample sizes will be found to satisfy the $\mathrm{M} \& \mathrm{~V}$ accuracy.

In the case study, the estimated sampling uncertainty is $C V_{R e f}=\{0.2,0.5\}$, which represents $c v_{1}=0.2$ and $c v_{2}=0.5$ in the two traffic light strata. In order to investigate flexibility and the model performance against different sampling uncertainties, the first simulations are carried out as follows: the optimal solutions obtained with $C V_{R e f}=\{0.2,0.5\}$ are taken as a reference. Then $C V_{\text {Ref }}$ is changed by $\pm 10 \%, \pm 20 \%$, and $\pm 50 \%$. The settings for 


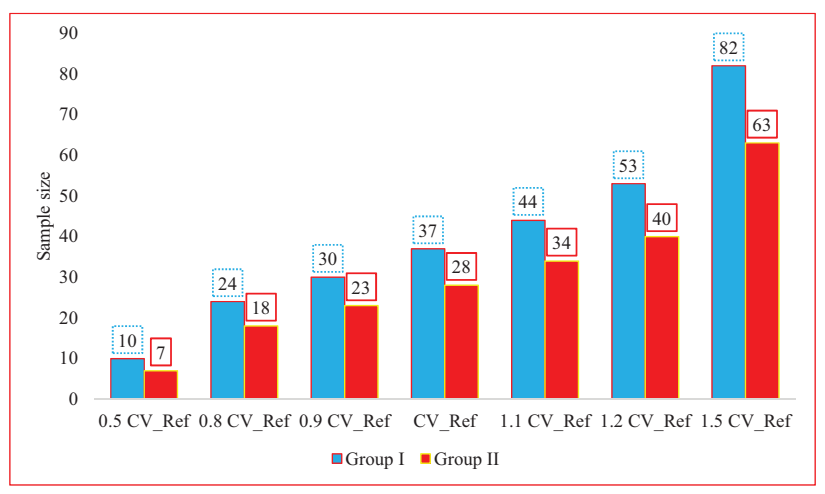

Figure 4: Sample size when sampling uncertainties change.

the optimisation are kept the same as given in the case study.

In the first simulation, the sample sizes namely, $n_{1}=37$ and $n_{2}=28$, are assigned to the two traffic light strata. When the sampling uncertainties change, the optimal accuracy levels of the baseline models are obtained and presented in Figure 3. It shows that when sampling uncertainty increases, more accurate models are required if the sampling efforts are limited. In the second simulation, the model accuracy namely, $C V m_{1}=5.356 \%$ and $C V m_{2}=9.233 \%$ are assigned to the two traffic light strata. When the sampling uncertainties change, the optimal sample sizes are obtained and presented in Figure 4. It shows that when sampling uncertainty increases, more sample sizes are required if the modelling efforts are limited.

From the simulation results, it is clear that a trade-off is possible between the modelling accuracy and the sampling accuracy. The proposed optimisation model is able to provide the $\mathrm{M} \& \mathrm{~V}$ practitioner the choices of having a more accurate baseline model with fewer sample sizes, or a less accurate baseline model with greater sample sizes to achieve the same $M \& V$ accuracy requirements.

\section{Conclusion}

A method to handle the major $\mathrm{M} \& \mathrm{~V}$ uncertainties; namely, the modelling and sampling uncertainties in M\&V costeffectively, has been developed. The proposed model offers optimal suggestions in allocating budget and efforts that need to be spent on handling the $\mathrm{M} \& \mathrm{~V}$ sampling and modelling uncertainties. This offers the $\mathrm{M} \& \mathrm{~V}$ practitioner an avenue to control the $\mathrm{M} \& \mathrm{~V}$ cost during the project planning phase. An optimal $\mathrm{M} \& \mathrm{~V}$ plan that satisfies the desired $\mathrm{M} \& \mathrm{~V}$ accuracy is designed using the proposed $M \& V$ cost minimisation model for a traffic light retrofit project as a case study. Results from the case study show that the optimal M\&V plan reduces the sampling cost by $42 \%$ and the total $\mathrm{M} \& \mathrm{~V}$ cost by $11 \%$ against the solutions obtained by partial optimisation, which demonstrates the effectiveness of the proposed model. The flexibility of the proposed model is also tested and revealed by the simulation results.

\section{Acknowledgement}

The authors would like to express our sincere appreciation to the editors and anonymous reviewers for their valuable comments and suggestions. This work is supported by the Centre of New Energy Systems and the National Hub for the Postgraduate Programme in Energy Efficiency and Demand Side Management at the University of Pretoria.

[1] Adcock, C. J. (1997). Sample size determination: a review. Journal of the Royal Statistical Society: Series D (The Statistician), 46, 261-283.

[2] Air Force Civil Engineer Support Agency (2011). Energy Measurement and Verification Reference Handbook. Technical Report, System Engineering and Management Corporation.

[3] ASHRAE (2002). ASHRAE Guideline 14: measurement of energy and demand savings. Technical Report, American Society of Heating, Refrigeration and Air Conditioning Engineers Atlanta, GA,

[4] Australian Energy Performance Contracting Association (2004). A best practice guide to measurement and verification of energy savings. Technical Report, Commonwealth of Australia.

[5] Aydinalp-Koksal, M., \& Ugursal, V. I. (2008). Comparison of neural network, conditional demand analysis, and engineering approaches for modeling end-use energy consumption in the residential sector. Applied Energy, 85, 271-296.

[6] Bertoldi, P., \& Rezessy, S. (2008). Tradable White Certificate schemes: fundamental concepts. Energy Efficiency, 1, 237-255.

[7] Burkhart, M. C., Heo, Y., \& Zavala, V. M. (2014). Measurement and verification of building systems under uncertain data: a Gaussian process modeling approach. Energy and Buildings, 75, 189-198.

[8] Carstens, H., Xia, X., \& Yadavalli, S. (2015). Measurement uncertainty and risk in measurement and verification projects. In International Enery Program Evaluation Conference. Long Beach, CA, USA.

[9] Carstens, H., Xia, X., \& Ye, X. (2014). Improvements to longitudinal clean development mechanism sampling designs for lighting retrofit projects. Applied Energy, 126, 256-265.

[10] Dalgleish, A. Z., \& Grobler, L. J. (2003). Measurement and verification of a motor sequencing controller on a conveyor belt. Energy, 28, 913-927.

[11] Department of Energy (2016). Post-2015 National Energy Efficiency Strategy. NOTICE 948 OF 2016, 40515, Department of Energy.

[12] Dong, B., Cao, C., \& Lee, S. E. (2005). Applying support vector machines to predict building energy consumption in tropical region. Energy and Buildings, 37, 545-553.

[13] Dong, B., Lee, S. E., \& Sapar, M. H. (2005). A holistic utility bill analysis method for baselining whole commercial building energy consumption in singapore. Energy and Buildings, 37, 167-174

[14] Escrivá-Escrivá, G., Roldán-Blay, C., \& Álvarez-Bel, C. (2014). Electrical consumption forecast using actual data of building end-use decomposition. Energy and Buildings, 82, 73-81.

[15] Eskom (2011). The measurement and verification guideline for demandside management proejcts. Technical Report, Eskom.

[16] Efficiency Valuation Organization (EVO) (2012). International performance measurement and verification protocol: concepts and options for determining energy and water savings. Technical Report.

[17] Giglio, T., Lamberts, R., Barbosa, M., \& Urbano, M. (2014). A procedure for analysing energy savings in multiple small solar water heaters installed in low-income housing in Brazil. Energy Policy, 72, 43-55.

[18] Granderson, J., \& Price, P. N. (2014). Development and application of a statistical methodology to evaluate the predictive accuracy of building energy baseline models. Energy, 66, 981-990.

[19] International Electrotechnical Commission (2003). International Standard 62053 part 21: Static meters for reactive energy (Classes 1 and 2). Technical Report.

[20] International Electrotechnical Commission (2003). International Standard 62053 part 23: Static meters for reactive energy (Classes 2 and 3). Technical Report.

[21] ISO/TC 301 Energy management and energy savings (2014). Energy management systems - Measurement and verification of energy performance of organizations - General principles and guidance. Technical Report, ISO 50015 ISO.

[22] Jayaweera, T., Haeri, H., Keeling, J., Khawaja, M. S., \& Rushton, J. (2013). The Uniform Methods Project: methods for Determining Energy 
Efficiency Savings for Specific Measures. Technical Report, The Cadmus Group Portland, Oregon.

[23] JCGM (2008). Evaluation of measurement data - guide to the expression of uncertainty in measurement. Technical Report ISBN. 50 JCGM Member Organizations Geneva.

[24] Kaiser, M. J., \& Pulsipher, A. G. (2010). Preliminary assessment of the louisiana home energy rebate offer program using IPMVP guidelines. Applied Energy, 87, 691-702.

[25] Ke, M., Yeh, C., \& Jian, J. (2013). Analysis of building energy consumption parameters and energy savings measurement and verification by applying equest software. Energy and Buildings, 61, 100-107.

[26] Lee, T., Liao, K., \& Lu, W. (2012). Evaluation of the suitability of empirically-based models for predicting energy performance of centrifugal water chillers with variable chilled water flow. Applied Energy, 93, 583-595.

[27] Minister of Finance (2013). Regulations in terms of section 12L of the Income Tax Act, 1962, on the allowance for energy efficiency savings. Government Notice 37136, National Treasury.

[28] Office of Environment \& Heritage (2012). Measurement and verification operational guide: best practice MEVV process. Technical Report, New South Wales Government.

[29] Pacific Northwest National Laboratory (2011). Metering best practices, a guide to achieving utility resource efficeincy. Technical Report, U.S. Department of Energy Oregon.

[30] The Regulatory Assistance Project (RAP) (2014). Energy Efficiency Evaluation, Measurement, and Verification: A Regional Review of Practices in China, the European Union, India, and the United States. Technical Report.

[31] Reddy, T., Saman, N., Claridge, D., Haberl, J., Turner, W., \& Chalifoux, A. (1997). Baselining methodology for facility-level monthly energy use - part 1: theoretical aspects. In ASHRAE Transactions (pp. 336-347). ASHRAE volume 103. (pt 2 ed.).

[32] Reddy, T. A., \& Claridge, D. E. (2000). Uncertainty of measured energy savings from statistical baseline models. HVAC R Research, 6, 3-20.

33] Reddy, T. A., Saman, N. F., Claridge, D. E., Haberl, J. S. et al. (1997) Baselining methodology for facility-level monthly energy use-part 2: Application to eight army installations. ASHRAE Transactions, 103, 348.

[34] Ridler, N., Lee, B., Martens, J., \& Wong, K. (2007). Measurement uncertainty, traceability, and the GUM. Microwave Magazine, IEEE, 8, 44-53.

[35] Sabapathy, A., \& Whittaker, J. (2011). An assessment of energy efficiency and carbon reductions from replacing halogen lamps with light emitting diodes in traffic lights a case study of Gloucestershire countys initiative. Energy and Environment, 22, 361-374.

[36] Stern, F., \& Vantzis, D. (2004). Protocols for evaluating energy efficiency - both sides of the Atlantic. IEPEC 2014 International Energy Policy and Programme Evaluation Conference.

[37] TecMarket Works (2004). The California evaluation framework. Technical Report, California Public Utilities Commission Oregon.

[38] Thompson, S. K. (2012). Sampling. (3rd ed.). New York: John Wiley \& Sons, Inc.

[39] UNFCCC (2009). General guidelines for sampling and surveys for smallscale CDM project activities. Technical Report,.

[40] UNFCCC (Version 02.0, 2012). Guideline for sampling and surveys for CDM project activities and programme of activities. Technical Report.

[41] Department of Energy (USA) (2008). MEVV guidelines: measurement and verification for federal energy projects. Technical Report.

[42] Vine, E., Kats, G., Sathaye, J., \& Joshi, H. (2003). International greenhouse gas trading programs: a discussion of measurement and accounting issues. Energy Policy, 31, 211-224.

[43] Walter, T., Price, P. N., \& Sohn, M. D. (2014). Uncertainty estimation improves energy measurement and verification procedures. Applied Energy, 30, 230-236.

[44] Xcel Energy (2013). Measurement and verification for retrofit and new construction projects. Technical Report, Xcel Energys 2009 Commercial Standard Offer Program Texas.

[45] Xia, X., \& Zhang, J. (2013). Mathematical description for the measurement and verification of energy efficiency improvement. Applied Energy, $111,247-256$

[46] Ye, X., \& Xia, X. (2016). Optimal metering plan for measurement and verification on a lighting case study. Energy, 95, 580-592.

[47] Ye, X., Xia, X., \& Zhang, J. (2013). Optimal sampling plan for clean development mechanism energy efficiency projects. Applied Energy, 112, 1006-1015.

[48] Ye, X., Xia, X., \& Zhang, J. (2014). Optimal sampling plan for clean development mechanism lighting projects with lamp population decay. Applied Energy, 136, 1184-1192. 